\begin{tabular}{|c|c|c|c|c|c|}
\hline \multirow{2}{*}{\multicolumn{2}{|c|}{ Prescriptions reviewed }} & Initial audit February 2010 & Re-audit September 2010 & Re-audit January 2012 & \multirow[b]{2}{*}{ Change } \\
\hline & & 384 & 387 & 387 & \\
\hline Standard & Target & & & & \\
\hline Prescriber identity & $100 \%$ & $18.4 \%$ & $95.2 \%$ & $98 \%$ & $+76.6 \%$ \\
\hline Prescriber contact & $100 \%$ & $7.8 \%$ & $77.9 \%$ & $84 \%$ & $+76.2 \%$ \\
\hline Additional chart(s) & $100 \%$ & $38.3 \%$ & $81.3 \%$ & $90 \%$ & $+51.7 \%$ \\
\hline Missed doses & $100 \%$ & $5.9 \%$ & $80.2 \%$ & $100 \%$ & $+94.1 \%$ \\
\hline
\end{tabular}

No conflict of interest.

\section{GRP-085 IDENTIFICATION OF RELEVANT DRUG INTERACTIONS IN NEONATAL INTENSIVE CARE UNITS}

doi:10.1136/ejhpharm-2013-000276.085

'A Cransac, ${ }^{2 D}$ Semama, ${ }^{3} \mathrm{~A}$ Lazzarotti, ${ }^{3} \mathrm{~J}$ Hugueny, ${ }^{4} \mathrm{C}$ Sgro, ${ }^{5} \mathrm{C}$ Ferdynus, ${ }^{6} \mathrm{JB}$ Gouyon, ${ }^{3}$ P Fagnoni. 'Saint Antoine Hospital (APHP), Pharmacy, Paris, France; '2Dijon University Hospital, Pediatrics, Dijon, France; 'Bijon University Hospital, Pharmacy, Dijon, France; ${ }^{4}$ Dijon University Hospital, Regional Center of Pharmacovigilance, Dijon, France; ${ }^{5}$ La Réunion University Hospital, Methodological Support Unit, La Réunion, France; ${ }^{6}$ La Réunion University Hospital, Neonatology, Saint-Pierre La Réunion, France

Background Among the different types of medication errors, drug interactions may have serious consequences in Neonatal Intensive Care Units (NICU). However, they can be easily detected with appropriate tools, particularly in the context of a computerised prescribing system with pharmaceutical analysis.

Purpose The objective of this study was to calculate a theoretical criticality index, using a method inspired by the Failure Modes, Effects and Criticality Analysis (FMECA) method for each drug interactions identified in NICU in order to prioritise them to pharmacists and physicians.

Materials and Methods The study was a retrospective review of prescriptions in a French NICU. The study included prescriptions for preterm infants with gestational age below 33 weeks and hospitalised between January 2006 and December 2009. For each prescription, drug interactions were evaluated with the French Theriaque ${ }^{\circledR}$ medication database. The criticality index of each drug interaction was calculated by multiplying occurrence, severity and detection scores. The scales of each score had been built by a multidisciplinary group. Severity and detection scores were assessed by pharmacists and physicians. Intraclass Correlation Coefficients (ICCs) were used to compare pharmacists' and physicians' scores, and a synthesis was realised.

Results Among the 907 prescriptions with at least 2 prescribed drugs (4605 prescriptions written, with 109 different drugs), 47 different drug interactions were identified with Theriaque ${ }^{\circledR}$. The 10 most critical drug interactions for pharmacists and physicians were detailed, and then a common medical and pharmaceutical synthesis was established. The ICC of detection was 0.75 (95\% CI: 0.63-0.88), and the severity was 0.32 (95\% CI: 0.08-0.56).

Conclusions This work highlights the importance of multidisciplinary collaboration in safe medication practise. This method can be used as a basis for future cooperation between medical teams and the pharmaceutical teams that make interventions. It is easily transferable to other medical specialties with the same objectives.

No conflict of interest.

\section{GRP-086 IDENTIFYING NEW TUBERCULOSIS CASES THROUGH PHARMACY DISPENSING RECORDS IN PROF DR FERNANDO FONSECA HOSPITAL, PORTUGAL}

doi:10.1136/ejhpharm-2013-000276.086

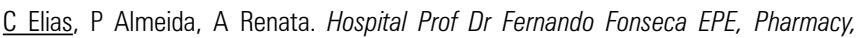
Amadora, Portugal
Background Controlling and preventing tuberculosis (TB) continues to be a major public healthcare challenge. Pharmacy and clinical records can thus contribute with important information concerning newly-diagnosed inpatients, treatment regimens and resistant strains.

Purpose To identify new tuberculosis (TB) cases through prescription records in a Portuguese General Hospital.

Materials and Methods This study took place in 2012, in Hospital Prof Dr Fernando Fonseca EPE (HFF), an 800-bed hospital. Patients were identified from Pharmacy dispensing records (Hosix v7.1 SIVSA) and clinical information was collected from the electronic medical records (Soarian Clinicals 3.1; Siemens). This data covered: age, sex, signs and symptoms, risk factors, outcomes of chest X-ray, diagnosis, respiratory isolates, therapeutic and microbiology results. Results To the end of September 201275 new cases of TB were identified. 38 diagnoses were made up to $24 \mathrm{~h}$ after hospital admission. The most frequent symptoms were non-productive cough $65.8 \%$, weight loss $55.3 \%$ and fever $50 \%$. There were 26 cases of pulmonary $\mathrm{TB}$ and 12 of extrapulmonary TB. 23 patients tested positive to the Ziehl Neelsen stain. 2 of the patients had resistant TB. 37 patients were diagnosed up to a maximum period of 10 weeks after hospital admission. The most frequent symptoms were nonproductive cough $40.5 \%$, weight loss $40.5 \%$ and fever $37.8 \%$. There were 16 cases of pulmonary TB, 13 extrapulmonary and 8 strictly clinical and imaging diagnoses. 3 patients tested positive to Ziehl Neelsen. 2 of the patients had resistant TB. By the time of the congress data will be updated for the year 2012 .

Conclusions The high rate of delayed-diagnosis TB contributes to an increase risk for the health care workers and other patients exposed to it. The hospital OHD used this study to demonstrate the importance of early diagnosis in the Emergency Department and faster microbiology results and of putting suitable isolation measures in place.

No conflict of interest.

\section{GRP-087 IMPACT OF AN ELECTRONIC MEDICINES RECONCILIATION PROGRAMME USED IN A GENERAL SURGERY UNIT}

doi:10.1136/ejhpharm-2013-000276.087

'A Giménez Manzorro, 'C Pérez Sanz, 'R Romero Jiménez, 2P Bodas Gutiérrez, ${ }^{2} \mathrm{MJ}$ Planelles López, ${ }^{3} \mathrm{R}$ Pla Mestre, ${ }^{4} \mathrm{JM}$ Bellón, 'A Herranz Alonso, 'M Sanjurjo Sáez. ${ }^{1}$ Hospital General Universitario Gregorio Marañon, Pharmacy Service, Madrid, Spain ${ }^{2}$ Hospital General Universitario Gregorio Marañon, General Surgery Service, Madrid, Spain: ${ }^{3}$ Hospital General Universitario Gregorio Marañon, Preventive Medicine Service, Madrid, Spain; ${ }^{4}$ Hospital General Universitario Gregorio Marañon, Statistics Service, Madrid, Spain

Background Medicines reconciliation is a key tool in the prevention of adverse drug events.

Purpose To assess the impact of a medicines reconciliation programme for hospital admission into a general surgery unit, including an electronic tool, in the number and type of unintended discrepancies between chronic medicines and medicines prescribed upon admission. 